\title{
Negative Fähigkeit nach W.R. Bion und die Balintarbeit - Aspekte bei Leiter und Teilnehmer
}

\section{Negative Capability according to W.R. Bion and Balint Group Work- Aspects concerning Leadership and Participants}

\section{()(1) $\odot \ominus$}

\section{Autoren \\ Ulrich Rüth ${ }^{1}$, Astrid Holch ${ }^{2}$}

Institut

1. Praxis für Kinder- und Jugendpsychiatrie und -psychotherapie und Institut der Dt. Akademie für Psychoanalyse, München

2. Klinik für Kinder- und Jugendpsychosomatik, Städt. München Klinik, München

\section{Schlüsselwörter}

Bion, Balintgruppe, Negative Fähigkeit, Containment, Prismatische Balintgruppe

\section{Key words}

Bion, Balint Group, Negative Capability, Containment, Prismatic Balint Group

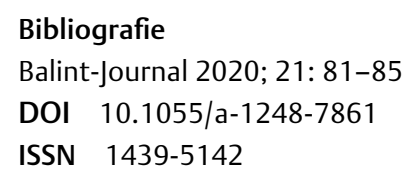
Creative Commons Attribution-NonDerivative-NonCommercial-License, permitting copying and reproduction so long as the original work is given appropriate credit. Contents may not be used for commercial purpose, or adapted, remixed, transformed or built upon. (https://creativecommons. org/licenses/by-nc-nd/4.0/)

\author{
Korrespondenzadresse \\ Dr. Ulrich Rüth \\ Facharzt für Kinder- und Jugendpsychiatrie und \\ -psychotherapie \\ Goethestraße 54 \\ 80336 München \\ Deutschland \\ ulrich.rueth@mnet-online.de
}

\author{
Astrid Holch \\ Oberärztin, Klinik für Kinder- und Jugendpsychosomatik \\ München Klinik \\ Kölner Platz 1 \\ 80804 München \\ astrid.holch@muenchen-klinik.de
}

\section{ZUSAMMENFASSUNG}

Negative Fähigkeit nach W.R. Bion zu verstehen als Aufnahmefähigkeit für Ungewusstes und Unbewusstes auf Seiten von Teilnehmern und Leitern stellt eine wichtige Voraussetzung für eine gelungene Balintarbeit dar. Das bisher wenig rezipierte Konzept ermöglicht ein vertieftes, tiefenpsychologisch-psychoanalytisches Erfassen der Phänomenologie von Balintarbeit. Hierzu gehören beobachtbare Widerstände oder Überforderungen von Teilnehmern, aber auch spezifische Techniken, wie die der Prismatischen Balintgruppe. Negative Fähigkeit zeigt Überschneidungen zum ebenfalls von Bion entwickelten Containment-Begriff, geht aber in den psychodynamischen Implikationen deutlich über diesen hinaus.

\begin{abstract}
Negative Capability, first used by Bion as a psychoanalytic term, signifying the capacity to take up and absorb unknown and unconscious aspects, is a prerequisite for leaders and participants to achieve a thriving Balint experience. Negative capability as a concept, yet not very commonly known, can help to understand the Balint phenomenology better in psychodynamic-psychoanalytic terms, such as resistance or overcharge in participants, but also special techniques like the Prismatic Balint Group. Negative Capabilty has overlaps with Bion's concept of containment, but shows deeper psychoanalytic implications.
\end{abstract}

Negative Fähigkeit nach Bion - Die Aufnahmefähigkeit für „Ungewusstes“

W.R. Bion, ein Schüler von Melanie Klein, geht mit seinen Konzepten und Überlegungen weit über ein übliches, klassisch-psychoanalytisches Denken hinaus. Für diejenigen, die eher wenig vertraut sind mit bionischem Denken, wirken sie gelegentlich befremdlich bis bizarr; allerdings stellen Bions Gedanken und Ideen eher eine Matrix dar, die zum Weiterdenken auffordert und von den „NachDenkenden “ aus der Abstraktion in Konkretes zurückübersetzt und damit angewendet werden muss. Bions Begrifflichkeiten des Containments [1,2] oder der Arbeitsgruppe [3] sind relativ gut rezi- 
piert und werden außerhalb kleinianisch-bionischer Zirkel weitreichend unproblematisch angewendet. Demgegenüber ist Bions Konzept der Negativen Fähigkeit (engl: Negative Capability) eher wenig aufgegriffen worden, wenngleich es hoch anregend erscheint, sich mit diesen Gedankenkonstrukt auseinanderzusetzen. Bion meint mit Negativer Fähigkeit eine Offenheit und die aktive Fähigkeit, Unsicherheit, Ambiguität, Widersprüchlichkeiten, Unverbundenheit und Nicht-Gewusstes aufzunehmen, also zu „containen“, aber darüber hinaus sich aktiv in einen entsprechenden Zustand des Aufnehmen-Wollens hinein zu versetzen, wie er günstigenfalls bei Therapeuten und in noch günstigerem Fall bei Patienten im Rahmen einer Psychotherapie vorliegen möge. Er entlehnte den Terminus der Negative Capability einem Brief des Dichters John Keats an dessen Bruder ${ }^{1}$. Keats sprach in seinem Brief von dem besonderen inneren Zustand des Dichters, der sich mit seiner künstlerischen Aufnahmefähigkeit Zweifeln, Geheimnissen und Unsicherheiten stellt, ohne nach Fakten und Gründen zu forschen. Einen ähnlichen Zustand sieht Bion für den Therapeuten² erstrebenswert an. Negative Fähigkeit ist nach Bion unabdingbar, um gemeinsam mit dem Patienten eine im therapeutischen Prozess dann tragfähige Veränderung zu erreichen. Sie ist gleichzeitig untrennbar verbunden mit der Idee, dass der psychoanalytische Psychotherapeut dem Patienten „ohne Gedächtnis und Wunsch“ begegnen sollte: Wenn möglich, vollständig unvoreingenommen und losgelöst von Verstrickungen, die in persönlichen Erinnerungen selbst an den Patienten - oder in unaufgelösten Gegenübertragungen begründet sind. Negative Fähigkeit meint im therapeutischen Beziehungskontext also, emotionale und mentale Zustände bei sich und dem Gegenüber wahrzunehmen und auszuhalten, und gleichzeitig, in reflektierendem Abwarten verbleibend, das Gesamtgeschehen zunächst nur zu beobachten und sich dann erst schrittweise zum Verstehen und zur Antwortfähigkeit vorzutasten.

Die Begrifflichkeit der Negativen Fähigkeit mag auch deshalb irritierend wirken, weil „negativ“ im üblichen Sprachgebrauch aversiv attribuiert wird. Bion verwendet in seinen Konzepten andernorts auch „+ “ (positiv) und „-“ (negativ), zu verstehen jeweils am ehesten im Sinn von „anwesend“ (+) und „abwesend“ (-). + K ( $K=$ "wissen wollen“) steht für die Anwesenheit des Prozesses von Wissen-Wollen, -K wäre zu verstehen als eine Entleerung von Wissen bzw. ein aktives Nicht-Wissen-Wollen.

Wenn Bion also von negativer Fähigkeit spricht, meint er die aktive Möglichkeit und Fähigkeit zur Aufnahme, vergleichbar mit einem Gefäß, das erst durch sein mögliches Leer-Sein überhaupt zu einem Behälter wird. Diese Aufnahmefähigkeit kann nun einfach vorhanden sein, oder wird durch innere Aktivität und eine innere Haltung hergestellt oder vergrößert. Negative Fähigkeit steht folglich Bions Konzept des Containments nahe, geht aber doch deutlich hierüber hinaus. Bion sieht in der Negativen Fähigkeit eine der wesentlichen Voraussetzungen gelingender (psychoanalytisch-)

$1 \quad \ldots$. is capable of being in uncertainties, mysteries, doubts, without any irritable reaching after fact and reason" Keats, J. (1970) The letters of John Keats: A selection. ed. Gittings, R., Oxford: Oxford University Press.pp 43.

2 Der besseren Lesbarkeit halber wird hier wie jeweils im weiteren Text die im Deutschen weiterhin übliche männliche Form genutzt, und auf Genderungen verzichtet. therapeutischer Arbeit: Das Eintreten neuer Einsichten - sowohl auf Seiten des Patienten wie auch des Therapeuten - hinge davon ab, dass Abstand davon genommen werde, eine durch Nicht-Wissen entstandene „Leere“ durch bloßes „Wissen“ (auf) zu füllen. Übersetzt bzw. übertragen würde dies dann einer intellektualisierenden Abwehr der tatsächlich vorliegenden, emotionalen Inhalte entsprechen - die wiederum eine Integration und Bearbeitung innerer Konflikte abwendet.

Die in der Begrifflichkeit einer negativen Fähigkeit transportierte Konnotation von etwas Aversiv-Negativem verhinderte möglicherweise eine stärkere Rezeption des Konzepts, insbesondere auch in der Sekundärliteratur. Das von Symington \& Symington [4] ausgearbeitete Grundlagenbuch zur klinischen Arbeit auf der Basis bionischen Denkens erwähnt Negative Capability nur am Rande und im New Dictionary of Kleinian Thought [5] wird sie vollständig ausgespart. Der eher knappe Eintrag im Dictionary of the Work of Bion [6] fokussiert bei der Erläuterung der Begrifflichkeit der Negativen Fähigkeit auf Neugier und Offenheit für Entdeckungen bei gleichzeitiger Fähigkeit, Unwissenheit auszuhalten. Betts [7] unterstreicht, dass Negative Fähigkeit eng verbunden sei mit der Haltung, dem Unbewussten zu vertrauen. Dies sei aber kein kontinuierlicher Zustand, sondern unterliege Fluktuationen. Gerade deswegen müssten wir nach Negativer Fähigkeit streben, also dem Unbewussten mehr trauen - sodass hier eine der wenigen vollständigen Paper aus psychoanalytischer Sicht verfügbar sind, die sich Bions Konzept widmen.

Einer schwachen Rezeption im genuin psychoanalytischen Therapieumfeld steht gegenüber, dass Negative Fähigkeit in der psychoanalytischen Organisationsberatung durchaus einen wichtigen Fokus darstellt: Manager müssen auf der Basis hoch unsicherer oder gar fehlender Informationen Entscheidungen treffen und dies zudem häufig in Situationen, die mit dem Tagesbewusstsein, also der intellektuell-begrifflichen Analyse nicht überblickbar sind [8]. Negative Fähigkeit im Zusammenhang der Leitung von Organisationen bedeutet, dass auf Unsicherheit beruhende Leitungsentscheidungen solange ausgehalten und durch den Leiter selbst „contained“ werden, bis der Entscheidungsträger durch die Gesamtsituation hinreichend „in-formed “3 wurde. So sind z. B. Entscheidungen zur Expansion eines Geschäftsbereichs abhängig von Entscheidungen konkurrierender Unternehmen, deren Produktentwicklungen, aber auch der konjunkturellen Marktentwicklung sodass erst im weiteren zeitlichen Verlauf ein Gesamtverständnis der bis dahin unsicheren Situation und der Implikationen des Ausgangs einer im übertragenen Sinn veranstalteten „Wette“ auf neuer Basis möglich wird [9]. Bions Konzept kann also sehr hilfreich sein, Leitung in Organisationen besser zu verstehen und Leitungskräfte zu unterstützen, die immanenten und z.T. extremen emotionalen Zustände der Unsicherheit begrifflich besser zu fassen und mit ihnen umzugehen. Negative Fähigkeit bedeutet auf Leitungsebe-

3 „in-formed“ lässt sich nicht treffend ins Deutsche übersetzen - gemeint ist hierbei die (innere) Modellierung und Formierung durch „In-formation“, also das Ersetzen der unmodellierten Unsicherheit durch fassbares Wissen. - Zu bedenken wäre hier ja auch, dass jede Spiegelung von Unsicherheit gegenüber einer Leitungsfigur am ehesten deren Abwehr induzieren wird und damit einen Rückzug auf scheinbar sicheres Terrain - mit der Folge größerer Fehleranfälligkeit in den Entscheidungen bei geringerem Einbezug intuitiver Aspekte. 
ne, aktiv Situationen von Unsicherheit und Nicht-Wissen gestaltend auszuhalten [10].

\section{Balintarbeit und „Negative Fähigkeit“}

In der Besprechung der Arzt-Patienten-Beziehung in der Balintgruppe wird dem fallvorstellenden Kollegen ein Containment angeboten [11], über das ungeordnete Affekte, Unsicherheiten, Wahrnehmungen und Beziehungskonstellationen - vergleichbar Bions $\beta$-Elementen - in denk- und bearbeitbare Vorstellungen und Ideen - Bions $\alpha$-Elemente - überführt werden $[12,13]$. In diesem Transformationsprozess nimmt die Gruppe bisher „Nicht-Gewusstes“ und „Nicht-Wahrgenommenes“ und bisher Verborgenes mittels projektiver Identifikation aus der Arzt-Patienten-Beziehung auf, um dieses „Unbewusste“ durch Bearbeitung im Gruppendiskurs in einen Bewusstseinsbereich zu bringen, wo es assimiliert, gefühlt und gedanklich erfasst werden kann. Anders als in der Supervisionsgruppe will die Balintarbeit keinen Algorithmus zum weiteren Vorgehen erarbeiten. Vielmehr soll die Fähigkeit gestärkt werden, auf den Patienten und seine Bedürfnisse weniger zu „reagieren“, sondern angemessener und freier zu „antworten“ [14]. Die Gruppe, ihr Leiter, wie auch jeder einzelne Teilnehmer in der Diskussion stellen damit ihre Aufnahmefähigkeit für Unsicherheiten zur Verfügung - und damit über ein bloßes Containment hinaus ihre „Negative Fähigkeit“. Die Gesamtgruppe verbleibt im günstigsten Fall während der gesamten Gruppensitzung im Zustand „negativer“, d. h. „offen gehaltener“ Kapazität, da keine konkret formulierte „Aktion“ angestrebt wird. Eine der wesentlichen Erfahrungen bei kontinuierlicher Balintarbeit ist eine nach der Gruppenarbeit erhöhte „Antwortfähigkeit“ auf den Patienten: Nach einer Vorstellung in der Gruppe treten regelmäßig eher unerwartete, neue Konstellationen in der nächsten Arzt-Patienten-Begegnung auf, die sich auf eine Veränderung des Arztes bzgl. des inneren Verstehens des Patienten und eine freiere Erwiderung auf dessen Nöte rückführen lassen.

Für gewöhnlich erleben sowohl der vorstellende Kollege als auch die Teilnehmer nach dem Diskussionsprozess in der Balintgruppe eine größere innere Leichtigkeit, welche vielleicht verstanden werden kann als eine innere Anfüllung mit produktiv zu nutzendem, seelischem Material. Dieser Vorgang geht mit einem inneren Bereicherungs- und Wachstumsprozess einher.

Der vorstellende und an der Gruppendiskussion nicht teilnehmende Kollege übt sich in seiner eigenen „negativen Fähigkeit“, indem er durch sein Zuhören die Möglichkeit erhält, von eigenen Reaktionen und handelndem Mitdiskutieren oder gar eventuellem Verneinen, Richtigstellen und Abwehren der dargebotenen gedanklichen und seelischen Inhalte abzusehen.

Die Gruppe stellt ihre „negative Fähigkeit“ zur Verfügung, indem sie über Wege der projektiven Identifikation die bisher unerkannten Anteile der Arzt-Patienten-Beziehung aufgreift und über die Gruppendiskussion sowohl dem emotionalen Vorbewussten wie dem Bewusstsein des Vorstellenden und zusätzlich auch den anderen Teilnehmern zugänglich macht. Aspekte einseitiger Aktion oder einer Evakuation unerträglicher Gefühle in die Gruppe werden bei gelingender Balintarbeit über den Gruppenprozess ausgeglichen - und über das „push-back“, also das Sich-Herausnehmen des fall- vorstellenden Kollegen aus dem Gruppenprozess, dokumentiert durch das leichte Zurücksetzen des Stuhls.

Der einzelne, sich in den Diskurs einbringende Teilnehmer öffnet sich im Gruppenprozess - wenn auch in unterschiedlicher Stärke - den angebotenen Diskussions-Bruchstücken und stellt dabei seine eigene Negative Fähigkeit als Container und Resonanzkörper für den Gruppenprozess zur Verfügung.

Leiter (und Co-Leiter) stellen durch Strukturgebung einen Rahmen her, über den beide wiederum sowohl ihre eigene Negative Fähigkeit wie die der Gruppe modellieren. Ihre Aufgabe ist es auch, bei zu großer Störung helfend einzugreifen, oder bei Überlastung von Containment und Negativer Fähigkeit Grenzen wiederherzustellen.

Eine Gruppenteilnehmerin wird durch die Beschreibung des Todes eines Kindes im geschilderten Fall emotional schwer mitgenommen, was ihr deutlich anzumerken ist. Die Co-Leitung schlägt ihr ein Verlassen des Raums vor. In der Zweiersituation außerhalb der Gruppe kann ein emotionales Containment für die Kollegin und deren Abgrenzungsfähigkeit wiederhergestellt werden. Zumindest vorläufig kann eine gewisse Aufnahmefähigkeit für den Gruppenprozess und die Fortsetzung der Teilnahme an der Gruppensitzung erreicht werden.

Abhängig von der Bereitschaft der Teilnehmer, sich auf emotionale Inhalte einzulassen, entsteht in der Balintgruppe ein unterschiedliches Niveau „Negativer Fähigkeit“.

Einem sehr rationalistischen, internistischen Kollegen fällt es schwer, sich auf den, emotionale Aspekte fokussierenden Gruppendiskussionsprozess einzulassen und zu beteiligen. Er sucht vorrangig nach Vorschlägen für konkretes Vorgehen und somatische Behandlungsstrategien. Bei der im Rahmen der Psychosomatischen Grundversorgung obligatorischen eigenen Fallschilderung werden verunsichernde, unklare und widersprüchliche Aspekte vermieden und weitgehend nur Fakten berichtet. Die sich in der Diskussion bemühende Gruppe und die von ihr benannten Fantasien und emotionalen Erlebnisse werden im Abschlussstatement des Kollegen letztlich entwertet und so die angebotenen Inhalte abgewehrt. Im bionischen Sinn hat der fallvorstellende Kollege eher keine Negative Fähigkeit wirksam werden lassen wollen oder können.

Wenn die „Negative Fähigkeit“ der Gruppe nicht ausreicht für die Aufnahme entscheidender Aspekte der vorgestellten Arzt-Patienten-Beziehung, kann im Verlauf der Gruppendiskussion das Einbringen dieser Aspekte durch den Leiter hilfreich werden. Besonders kann dies der Fall sein bei parasitären Arzt-Patienten-Beziehungen, die ohnehin nicht der üblichen Beziehung zwischen einem Patienten und einem Helfendem entsprechen. Hier ersetzt der Leiter dann ggf. durch eigenes Benennen des parasitären Modus die blockierte Negative Fähigkeit der Gruppe [15]. Vorsicht scheint dabei geboten, um die Gruppe nicht durch intellektualisierende Angebote im emotionalen Prozess zu bremsen.

Einige Balintleiter bevorzugen „freiwillige“ Gruppen - im Gegensatz zu jenen Ausbildungsgruppen, deren Teilnehmer sich auf- 
grund verpflichtender Curricula im Rahmen der Psychosomatischen Grundversorgung oder der Facharztausbildung zusammenfinden müssen. Bei „freiwilligen“ Teilnehmern kann - auch aufgrund deren für gewöhnlich vorliegender längeren Balinterfahrung - von einer größeren inneren Bereitschaft zur emotionalen Teilnahme und Sich-dem-Gruppenprozess-Überlassen ausgegangen werden, und damit von einer größeren „negativen Fähigkeit“ , die dann auch mehr Lebendigkeit in der Gruppendiskussion zur Folge hat.

\section{Prismatische Balintgruppen - eine „Technik“ zur Erhöhung Negativer Fähigkeit}

Unterschiedliche Ansätze versuchen im Rahmen der Balintarbeit einen erweiterten Zugang zu emotionalen Zuständen zu erreichen, wie z. B. Psychodrama-Balintgruppen [16] oder Balintgruppen mit Aufstellungsarbeit. In der von Drees konzipierten Prismatischen Balintgruppe [17] wird Negative Fähigkeit spezifisch beübt: Die Gruppe fokussiert - als Weiterentwicklung des klassischen Balintkonzepts - auf Stimmungsprozesse, körperlich-sinnliche Beschreibungen und frei flottierende Fantasien, die alle ausdrücklich nicht auf die Arzt-Patienten-Beziehung hin oder auf eine dort vermutete Störung gedeutet werden. Drees geht davon aus, dass die Transformation von gerichteten Beziehungsgefühlen in „sinnliche und stimmungsbezogene Gefühle des Selbsterlebens “ ganzheitliche, sinnsuchende und sinnlich-gestimmte Kommunikationsprozesse möglich macht. In der konkreten Anwendung der prismatischen Methode wird auf Detailbeschreibungen von fantasierten Bildern und Inhalten Wert gelegt. Angesprochene Stimmungen und Fantasien werden nicht in Richtung ihrer symbolischen Bedeutung diskutiert.

Die Teilnehmer werden angehalten, sich möglichst ohne jegliche innere Zensur auf auftretende Fantasien und Körperempfindungen einzulassen und diese in die Gruppe einzubringen. Insgesamt handelt es sich bei der Prismatischen Balintgruppe nach Drees um eine „sinnlich imaginative Arbeitsmethode“.

Legt man Bions Konzept der Negativen Fähigkeit zugrunde, so wird diese Negative Fähigkeit als nicht-zensierende Aufnahmefähigkeit genutzt für die Evozierung zunächst scheinbar ungeordneter Fantasien und Stimmungen bei gleichzeitiger Erweiterung gerade dieser Negativen Fähigkeit. Nutzung und Erweiterung der $\mathrm{Ne}$ gativen Fähigkeit geschehen über das Einbringen von intellektuell Nicht-Fassbarem, bisher Nicht-Gedachtem und „Nicht-Gewusstem“ in Gestalt unzensierter Affektbruchstücke, Körperwahrnehmungen und scheinbar abwegiger Fantasien. All dieses durchaus verwirrende und verunsichernde Material wird in den containenden Gruppenprozess eingebracht und erweitert dabei den Zugang zum Vorbewusstem. Folgt man den Schilderungen Drees ${ }^{4}$, so werden über die Prismatische Balintgruppe in überraschender Weise neue Möglichkeiten in der Beziehungsgestaltung angestoßen, weil über einen vorrangig nicht intellektuellen Prozess zuvor nicht greifbare Inhalte energetisch aufgeladen wurden.

\section{Negative Fähigkeit und Ergebnisse der Forschung zur Balintarbeit}

Die Forschung zur Wirksamkeit von Balintgruppen hatte bisher sehr unterschiedliche Foci. Sie kam zu einigen, hier herausgegriffenen und ätiologisch nur bedingt erklärbaren Ergebnissen: So wurde eine Verbesserung der Empathiefähigkeit $[18,19]$ aufgezeigt, aber z. B. auch eine Veränderung im Kommunikationsverhalten. Diese zeigt sich u. a. in der Bereitschaft, den eigenen Gesprächsanteil zu reduzieren und mehr zuzuhören [20]. Bei Ärzten mit somatischer Ausbildung bewirkte die Teilnahme an einer Balintgruppe eine verstärkte Auseinandersetzung mit interpersonellen Phänomenen der Arzt-Patient Beziehung und mit Übertragungsdynamiken [21].

Nimmt man als Ausgangspunkt Bions Konzept Negativer Fähigkeit, welche in der Balintgruppe beübt wird, so ließen sich die angeführten Ergebnisse recht zwanglos erklären: Die Einfühlung in ein Gegenüber wächst, wenn sich Offenheit für Fremdes und Unverständliches vergrößert. Je mehr ich aufzunehmen bereit bin, umso weniger werde ich mich mit meinen eigenen emotionalen oder auch intellektualisierenden Inhalten in ein Gespräch mit dem Patienten einbringen müssen - analog Bions „Zuhören ohne Gedächtnis und Wunsch“. Wenn ich aus dem somatischen, und damit eher funktionalen Bereich der Medizin komme, weitet sich in der Balintarbeit meine Negative Fähigkeit, also meine Aufnahme und intuitives Verstehen von interpersonalen und emotionalen Aspekten meiner Patientenbegegnung.

Abhängig vom Forschungsansatz lässt sich in unterschiedlicher Weise ein Zusammenhang herstellen zwischen qualitativen und quantitativen Ergebnissen auf der einen Seite mit einer wachsenden Fähigkeit der Teilnehmer auf der anderen Seite, sich einer emotional unsicheren Situation zu stellen und mit dieser konstruktiv und eben nicht abwehrend-wertend umzugehen, und damit mit einer größeren Negativen Fähigkeit nach Bion.

\section{Ausblick}

Benötigen wir Bions Konzept Negativer Fähigkeit? In der psychoanalytischen Organisationsberatung wird diese Gedankenfigur genutzt, um Angstkomponenten in unübersichtlichen Situationen zu erklären, zu „containen“ und durch konstruktiven Umgang zu verringern. In der praktischen Balintarbeit könnte mithilfe des Konzepts der Negativen Fähigkeit ein unterschiedliches „Ansprechen“ der Teilnehmer auf die Gruppenarbeit besser verstehbar werden: Hier spielt die entscheidende Rolle das unterschiedliche Ausmaß, wie weit sich die einzelnen Gruppenmitglieder gegenüber unsicheren, ambivalenten und diffus-emotionalen Aspekten der Arzt-Patienten-Beziehung öffnen können und wollen. Es könnte fruchtbar sein, Strategien zu entwickeln, eine Erweiterung der Negativen Fähigkeit bei potentiellen Interessenten und Teilnehmern zu stärken und sie so für die kontinuierliche Balintarbeit zu gewinnen.

4 oder eigenen Erfahrungen in Prismatischen Balintgruppen, sei es als Teilnehmer oder Leiter. 


\section{Autorinnen/Autoren}

\section{Ulrich Rüth}

Dissertation an der Psychosomatik. 18 Jahre psychodynamisch orientierte Oberarzttätigkeit in der Kinder- und Jugendpsychiatrie. 2011 Praxisniederlassung.

\section{Astrid Holch}

Nach Weiterbildung zur Fachärztin für Kinder- und Jugendpsychiatrie und -psychotherapie. 5 Jahre Tätigkeit in einer sozialpsychiatrischorientierten Praxis, jetzt Oberärztin in der Kinder- und Jugendpsychosomatik.

\section{Interessenkonflikt}

Die Autoren sind Mitglied der Dt. Balintgesellsachaft. Sie erzielen Einnahmen als Balintgruppenleiter.

\section{Literatur}

[1] Bion WR. Attacks on linking. International Journal of Psychoanalysis 1959; 308-315. Erneut publiziert in Bion WR. Second Thoughts. London: Heinemann; 1967

[2] Bion WR. Attention and Interpretation. London: Tavistock; 1970

[3] Bion WR. Erfahrungen in Gruppen. Stuttgart, Klett-Cotta; Frankfurt/ Main, Fischer; 1971. [Engl.: Experiences in groups. London: Tavistock; 1961]

[4] Symington J, Symington N. The clinical thinking of Wilfred Bion. London: Routledge; 2001

[5] Bott Spilius E, Milton J, Garvey P, Cove C, Steiner D. New Dictionary of Kleinian Thought. London: Routledge; 2011

[6] Lopez-Corvo RE. The Dictionary of the Work of Bion. London: Karnac; 2003

[7] Betts W. (2009). Negative capability. Psychoanalysis downunder, 7B. Online Journal Australian Psychoanalytic Society 2009; https://drive. google.com/file/d/1iDH7yG4AbAZLIH_7Tzd9DYrIXOfvhwUU/view Accessed 13.03.2020

[8] French R, Simpson P, Harvey C. Negative capability. A contribution to the understanding of creative leadership. In: Sievers, B., Brunning, H., De Gooijer, J. and Gould, L. eds.: Psychoanalytic Studies of Organizations: Contributions from the International Society for the Psychoanalytic Study of Organizations. London: Karnac; 2009
[9] French R. „Negative capability”: managing the confusing uncertainties of change. Journal of Organizational Change Management 2001; 4: 480-492

[10] Ou L. Keats and negative capability. London: Continuum International Publishing Group; 2001

[11] Rüth U, Holch A. Die Balint-Gruppe als Container für die Not des Arztes. Dynamische Psychiatrie 2012; 45: 78-91

[12] Rüth U. Classic Balint Group work and the thinking of W.R. Bion: How Balint work increases the ability to think one's own thoughts. Group Analysis 2009; 42: 380-391

[13] Rüth U. Wichtige Konzepte Bions und Gedanken zur Balintgruppenarbeit. Balintjournal 2005; 7: 43-48

[14] Symington N. The possibility of human freedom and its transmission (with particular reference to the thought of Bion). Int J PsychoAnalysis 1990; 71: 95-106

[15] Rüth U. Parasitäre Beziehungen und aktives Leiterverhalten in der Balintgruppe - mit besonderem Bezug zu den theoretischen Annahmen von W. R. Bion. Balintjournal. 2019; 20: 75-78. doi: http:// dx.doi.org/ 10.1055/a-0978-3926

[16] Bachmann JP. An Introduction to Balint-Psychodrama. Journal of the Balint Society 2015; 43: 5-12

[17] Drees A. Prismatische Balint-Gruppen. Balint Journal 2001; 2: 69-73

[18] Kutter P. Empathietraining in Balint-Gruppen: Forschung mit Psychologiestudenten. In: Körner J, Neubig H, Rosin U. (Hg): Die Balintgruppe in Klinik und Praxis. Berlin: Springer-Verlag; 1990: 167-180

[19] Cataldo KP, Peeden K, Geesey ME, Dickerson L. Association between Balint training and physician empathy and work satisfaction. FamMed 2005; 37: 328-331

[20] Obliers R, Köhle K, Kaerger H, Faber ], Koerfer A, Mendler T, Waldschmidt DT. Video-Dokumentation als Instrument der Qualitätssicherung: Evaluation der Entwicklung ärztlichen Gesprächsverhaltens nach Balint-Gruppenteilnahme. In: Bahrs O, Fischer-Rosenthal W, Szecsenyi J Vom Ablichten zum Im-Bilde-Sein. Ärztliche Qualitätszirkel und Video-Analysen Würzburg: Königshausen und Neumann; 1996: 261-290

[21] Flatten G, Möller H, Aden J, Tschuschke V. Die Arzt-Patientenbeziehung gestalten: Wie nützlich sind Balintgruppen und für wen? Zeitschrift für Psychosomatische Medizin und Psychotherapie 2017; 63: 267-279. doi: https://doi.org/10.13109/zptm.2017.63.3.267 